\title{
Palm Vein Pattern Visual Interpretation Using Laplacian and Frangi-Based Filter
}

\author{
Zarina Mohd Noh*1, Abdul Rahman Ramli², Marsyita Hanafi ${ }^{3}$, M Iqbal Saripan ${ }^{4}$, Ridza Azri Ramlee \\ 1,2,3,4 Department of Computer and Communication Systems Engineering, Faculty of Engineering, \\ Universiti Putra Malaysia, 43400 UPM Serdang, Selangor, Malaysia \\ ${ }^{1,5}$ Fakulti Kejuruteraan Elektronik dan Kejuruteraan Komputer, Universiti Teknikal Malaysia Melaka (UTeM), \\ Hang Tuah Jaya, 75450 Durian Tunggal, Melaka, Malaysia
}

\begin{tabular}{l}
\hline \hline Article Info \\
\hline Article history: \\
Received Nov 15, 2017 \\
Revised Jan 21, 2018 \\
Accepted Feb 11, 2018 \\
\hline
\end{tabular}

Keywords:

Frangi-based filter

Image processing

Laplacian filter

Near infrared palm image

Palm vein pattern detection

\begin{abstract}
Detection of palm vein pattern through image processing techniques is an open problem as performance of each technique is closely related to the sample image gathered for the processing. The detected palm vein pattern is useful for further analysis in biometrics application and medical purpose. This paper aims to investigate the application of Laplacian filter and Frangibased filter in detecting vein pattern contained in a near infrared illuminated palm image. Both filtering techniques are applied independently to two palm image databases to compare their performance in translating vein pattern in the image visually. Through empirical study, it is observed that Laplacian filter can translate the vein pattern in the image effectively. But preprocessings involved before the application of Laplacian filter need to be performed to accurately translate the vein pattern. The implementation of Frangi-based filter, while simplifying the detection process without the need of extra pre-processing, resulted in only certain vein pattern detected. Using pixel-by-pixel objective assessment, the rate for Laplacian filter in detecting vein pattern are generally more than $85 \%$ compared to Frangi-based filter; where it ranges from $60 \%$ to $100 \%$.
\end{abstract}

Copyright $@ 2018$ Institute of Advanced Engineering and Science. All rights reserved.

\section{Corresponding Author:}

Zarina Mohd Noh,

Fakulti Kejuruteraan Elektronik dan Kejuruteraan Komputer, Universiti Teknikal Malaysia Melaka (UTeM),

Hang Tuah Jaya, 75450 Durian Tunggal, Melaka, Malaysia.

Email: zarina.noh@utem.edu.my

\section{INTRODUCTION}

Palm image captured by the help of near infrared (NIR) illumination contained palm vein pattern information which is unseen through direct human visualization. The vein pattern can be unveiled using various combination of image processing techniques [1], depending on requirements of the extraction process. Direct translation of palm vein pattern from a NIR palm image is useful especially in biometrics and medical application. In biometrics, palm vein pattern for each person had been proven to have high distinction making it one of favourable modality for identification [2], [3]. In medical, visualization of vein pattern is useful for ease of venipuncture process and educational purpose [4], [5].

Previous works on vein pattern mostly covered the extraction of vein pattern acquired from the back-of-hand [6], [7], fingers and wrist area [8], for biometric matching performance but not for vein visualization purpose. In the case of back-of-hand vein pattern extraction, a group of image processings had been done to detect the vein pattern which are gray level normalization, Gaussian and median filtering (noise reduction), NiBlack method (segmentation) and thinning [6]. In other works, 2-D Gaussian filter had been used for noise reduction, in addition to image normalization, local adaptive thresholding and thinning algorithm for back-of-hand vein pattern extraction [9]. Gaussian and median filtering had also been reported 
to be used for back-of-hand vein extraction in other works, in addition to segmentation and skeletonization algorithm [10]. In finger and wrist vein extraction, three different segmentation approach that are active contours, Otsu thresholding and Frangi-based filter had been tested in a work that compares the biometric matching performance [8]. In wrist vein extraction, a group of image pre-processings had been implemented to detect the vein pattern using adaptive non-local means, nonlinear diffusion algorithm (noise reduction and edge enhancement), and Frangi-based filter [11]. None of the mentioned works addressed the vein pattern visual interpretation directly as the highlight in the work is on the biometric matching performance.

Although there are numerous filters and techniques available for vein pattern extraction, this paper compares only two of the filtering methods that can visually extract palm vein pattern from a NIR palm image. The two filtering methods are Laplacian filter [12] and Frangi-based filter [13]. The two filters are chosen based on their difference in operation, in which the first one aims to detect edges in an image, while the latter had been specifically developed to detect vein pattern in medical-related images. These two filters are compared because of its ease of implementation in extracting vein pattern, besides its straight-forward operation in extracting line and edges in an image. With the aim to extract palm vein pattern visually, the approach in executing both filtering techniques on NIR palm images will be demonstrated in this paper.

\subsection{Laplacian Filter}

Laplacian filter is one of edge detection processing that is commonly used for image segmentation. It detects edges by finding second-order derivation of an image function [14]. Edges in an image will be detected if there is any extreme shifts between the neighbouring pixel values. The second-order derivative for Laplacian filter is calculated by equation (1) as follows:

$$
\begin{aligned}
& \mathrm{L}_{\mathrm{I}}(\mathrm{x}, \mathrm{y})=\frac{\partial^{2} \mathrm{I}}{\partial^{2} \mathrm{x}}+\frac{\partial^{2} \mathrm{I}}{\partial^{2} \mathrm{y}} \\
& \text { Where: } L_{I}(x, y) \quad \text { = Laplacian value of a pixel at location }(\mathrm{x}, \mathrm{y}) \text { in image } I \\
& \text { I }=\text { Input image }
\end{aligned}
$$

\subsection{Frangi-based Filter}

Frangi-based filter or also known as Frangi vesselness filter, is originally designed for filtering vein pattern in two-dimensional X-ray medical images and three-dimensional volumetric magnetic resonance angiography images [15]. It is derived from eigenvalues of Hessian matrix analysis in measuring the local orientation to interpret vascular geometric properties of an image as in equation (2) [15]. Frangi-based filter is initially developed for vein pattern that appear as bright structure in dark background. Details for each parameter in equation (2) are as discussed in its implementation for forearm vascular pattern recognition purpose [13].

$$
\begin{aligned}
\mathrm{F}_{\mathrm{I}}(\mathrm{s})= & \left\{\begin{array}{ll}
\exp \left(\frac{\mathcal{R}_{\mathcal{B}}{ }^{2}}{2 \beta^{2}}\right)\left(1-\exp \left(-\frac{\mathcal{S}^{2}}{2 \mathrm{c}^{2}}\right)\right), & \text { if } \lambda_{2}<0
\end{array} \quad\right. \text { otherwise } \\
\text { Where: } \quad \begin{array}{cl}
F_{I}(s) & =\text { Frangi-based measure of vessel-like features in image } I
\end{array} \mathcal{R}_{\mathcal{B}} & =\frac{\lambda_{1}}{\lambda_{2}}, \text { eigenvalues / blobness measure in two-dimensional image } \\
\mathcal{S} & =\text { second-order structureness of Hessian matrix norm } \\
\beta, c & =\text { thresholds for filter sensitivity control }
\end{aligned}
$$

\section{IMAGE FILTERING IMPLEMENTATION}

A total of 2400 NIR palm images are used for the filtering implementation executed by OpenCVPython environment [16]. Both filtering process are implemented on two NIR palm image databases that are obtained from the Chinese Academy of Sciences' Institute of Automation (CASIA) [17] and the Hong Kong Polytechnic University (PolyU) [18]. The two databases information are as detailed in Table 1.

Table 1. Detail of Palm Image Databases Used for Filtering Demonstration.

\begin{tabular}{ccccc}
\hline Database & Number of Subjects & Hand/Session & Number of Samples & Total Images \\
\hline CASIA & 100 & Right-hand & 6 & 600 \\
& \multirow{2}{*}{100} & Left-hand & 6 & 600 \\
PolyU & First-session & 6 & 600 \\
& & Second-session & 6 & 600 \\
\hline
\end{tabular}


Laplacian filtering is performed following the execution of a set of image pre-processings as illustrated in Figure 1. This is because, direct implementation of Laplacian filter on raw palm images will reveal extra information in the image such as palm print, palm lines and palm creases besides the palm vein pattern. The image pre-processings will ensure that only palm vein pattern will be highlighted in the palm image. The image pre-processings shown in Figure 1 are extraction of Region-of-Interest (ROI), Contrast Limited Adaptive Histogram Equalization (CLAHE), bilateral filter and morphological dilation operation. Each of these pre-processing has its own parameters that requires adjustment to ensure its applicability in enhancing the image contrast and reducing noise for the implementation of Laplacian filter later on.

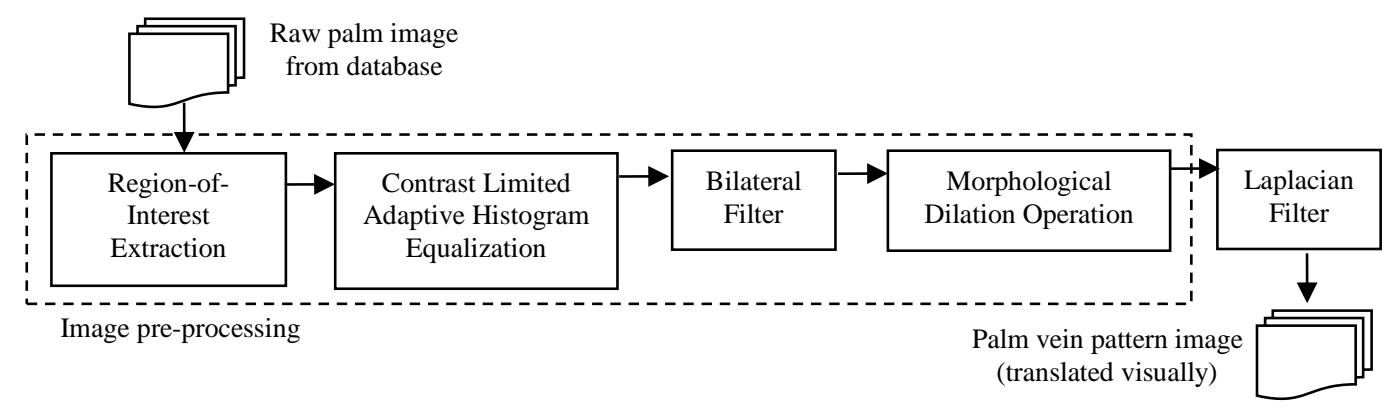

Figure 1. Laplacian Filtering Implementation Steps

On the other hand, Frangi-based filter is executed on raw palm images directly after an ROI extraction process as shown in Figure 2. This is because, additional image pre-processings as in Figure 1 will distort the extraction of vein pattern by the application of Frangi-based filter later on. Besides, derivation of Frangi-based filter is motivated by the intention to extract vein pattern from medical images. Its derivation had already included the image contrast and noise reduction techniques in the formula. Hence, the implementation steps for Frangi-based filter in extracting palm vein pattern had been reduced to the processes as illustrated in Figure 2. In terms of computational cost, Frangi-based filter is economical than Laplacian filter implementation as it does not require extra image pre-processings.

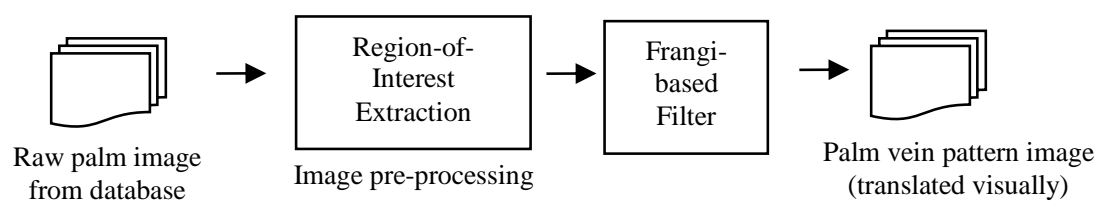

Figure 2. Frangi-based Filtering Implementation Steps

\section{RESULTS AND ANALYSIS}

Outcomes of the image filtering are as demonstrated in Section 3.1. Section 3.2 discusses the results of filtering implementation by comparing the rate of similarity through pixel-by-pixel detection of palm vein pattern detected.

\subsection{Filtering Demonstration}

The implementation of filtering processes resulted in sample outcomes as compiled in Figures 3-6. The raw palm images in both Figures 3 and 4 are chosen from the same subject to compare the translated vein pattern after the filtering process. It can be seen that both filtering process are able to detect palm vein pattern visually from the CASIA's NIR palm image, but the difference lies in the types of vein pattern extracted. While most vein pattern is detected through Laplacian filter, Frangi-based filter mostly detect large-sized vein pattern in the image. 


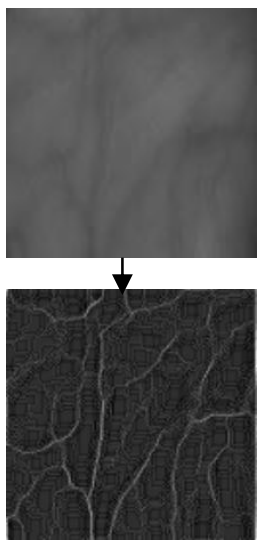

(a) Random Sample 1
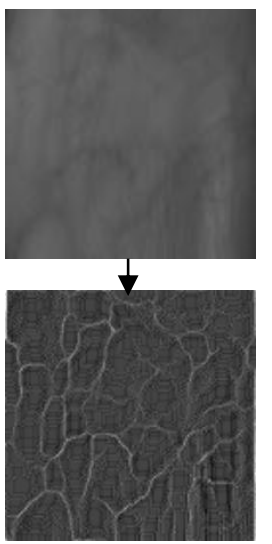

(b) Random Sample 2
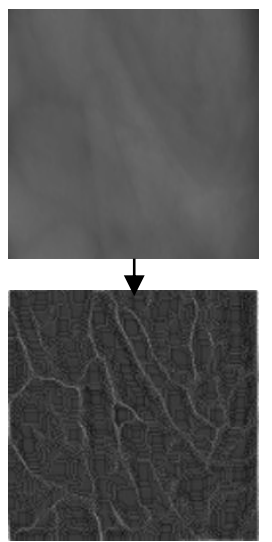

(c) Random Sample 3

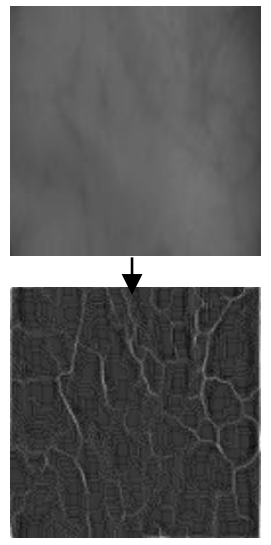

(d) Random Sample 4

Figure 3. Palm Image from CASIA Database Before and After the Implementation of Laplacian Filter

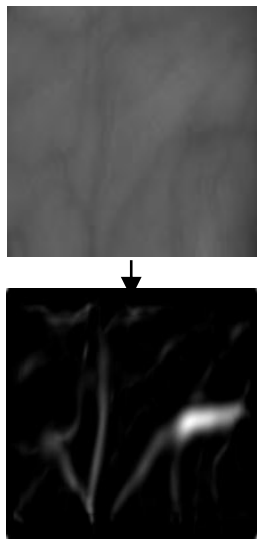

(a) Random Sample 1

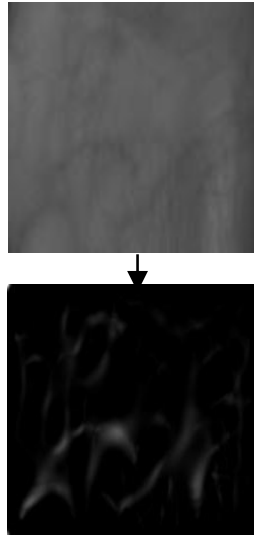

(b) Random Sample 2

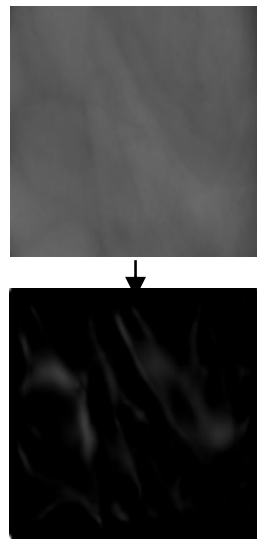

(c) Random Sample 3

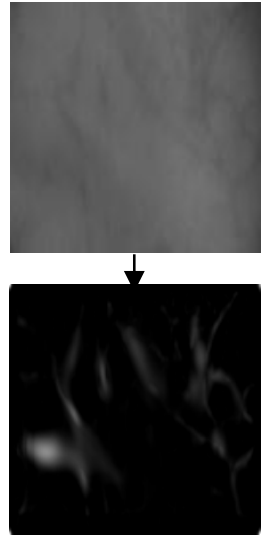

(d) Random Sample 4

Figure 4. Palm Image from CASIA Database Before and After the Implementation of Frangi-based Filter

Observing the translated vein pattern from PolyU's NIR palm image in Figure 5 and 6, its performance is as spotted in CASIA database. Most vein pattern in Figure 5 can be detected visually by Laplacian filter implementation, while Frangi-based filter mostly detect large-sized vein pattern in the image (Figure 6). Both Figures 5 and 6 use the same raw palm image from PolyU database to compare the filters' ability in translating the vein pattern visually. 


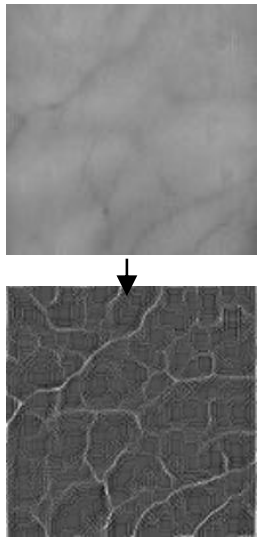

(a) Random Sample 1
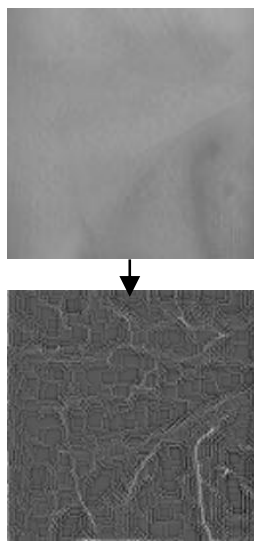

(b) Random Sample 2

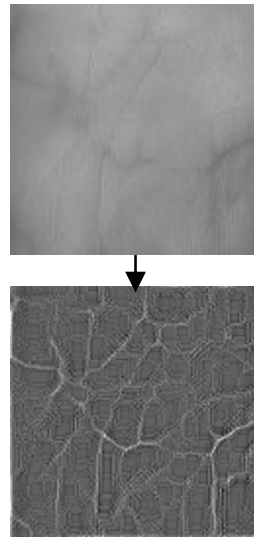

(c) Random Sample 3

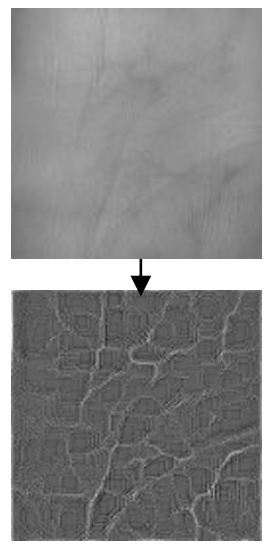

(d) Random Sample 4

Figure 5. Palm Image from PolyU Database Before and After the Implementation of Laplacian Filter
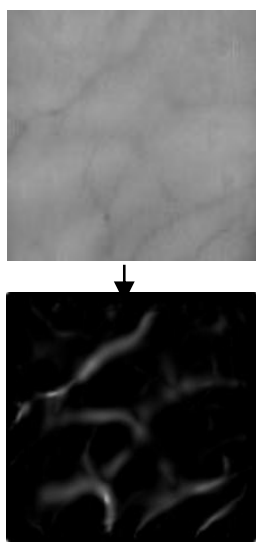

(a) Random Sample 1

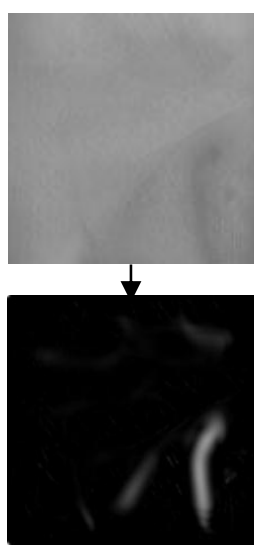

(b) Random Sample 2

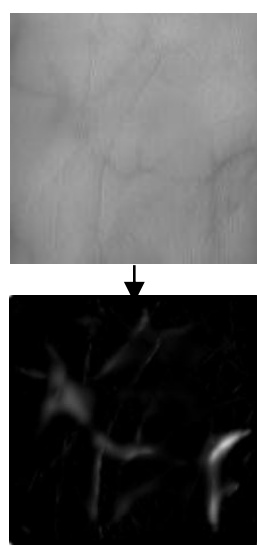

(c) Random Sample 3

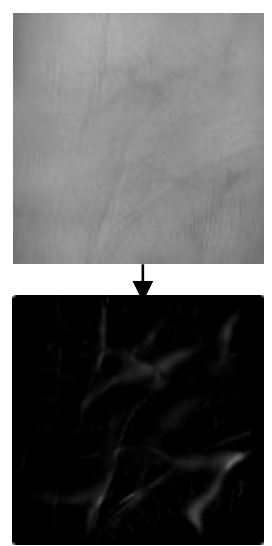

(d) Random Sample 4

Figure 6. Palm Image from PolyU Database Before and After the Implementation of Frangi-based Filter

\subsection{Discussion and Analysis}

To further illustrates the performance of both filtering techniques in detecting palm vein pattern, a pixel-by-pixel comparison of the detected vein pattern is done with a reference palm vein pattern images. The pixel-by-pixel comparison is chosen as the assessment method to check if the same vein pattern had been extracted every time the filtering techniques are implemented on the vein image. The reference palm vein pattern for each subject (100 subjects) is constructed by averaging the detected palm vein pattern image (six samples per subject) using each filtering technique. The comparison rate are as presented in Figures 7-10 for the six samples of each subject. While the similarity rate for vein pattern extracted by Laplacian filter ranges around $90-97 \%$ for CASIA database (Figure 7), the similarity rate for Frangi-based filtered image ranges around $70-97 \%$ (Figure 8). 


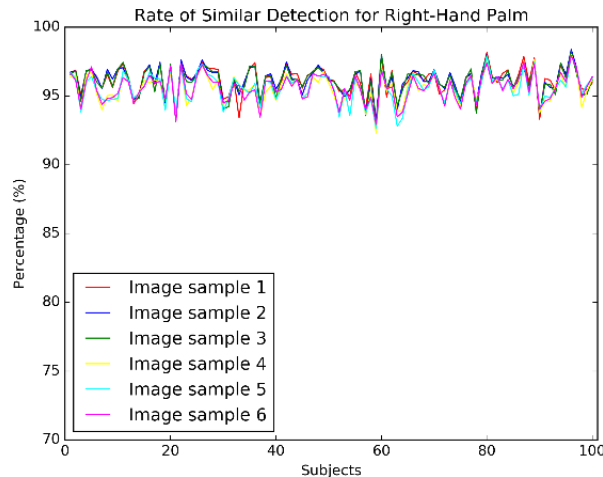

(a)

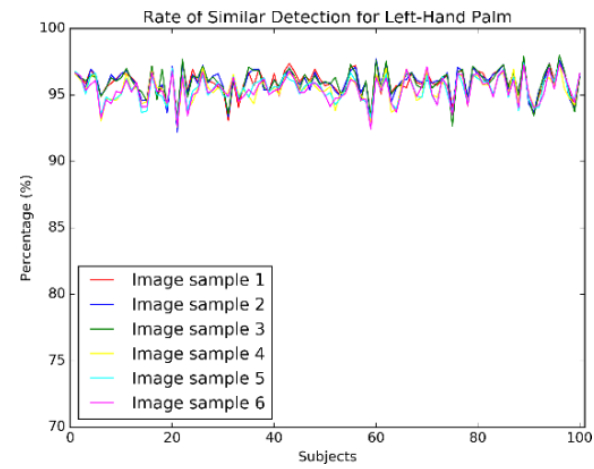

(b)

Figure 7. Rate of Similarity for Pixel-by-Pixel Detection of CASIA Database After Implementation of Laplacian Filter for (a) Right-Hand Palm, and (b) Left-Hand Palm

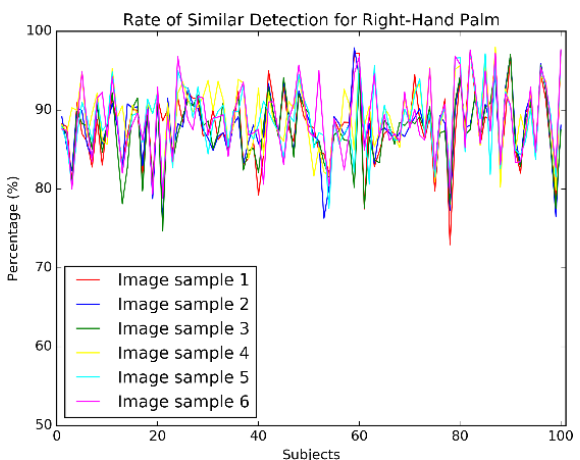

(a)

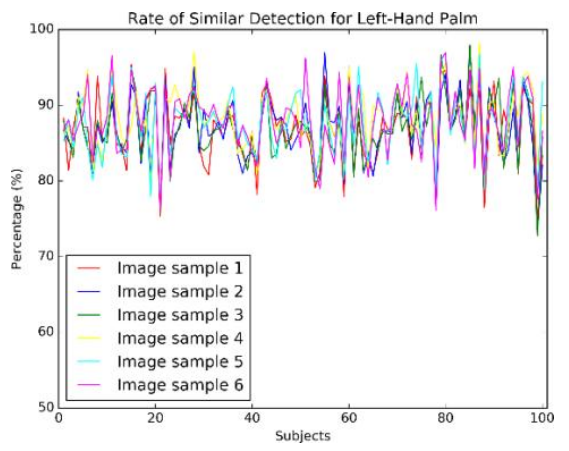

(b)

Figure 8. Rate of Similarity for Pixel-by-Pixel Detection of CASIA Database After Implementation of Frangi-Based Filter for (a) Right-Hand Palm, and (b) Left-Hand Palm

The similarity rate for PolyU database ranges around $85-95 \%$ for Laplacian filtered image (Figure 9), while for Frangi-based filtered image; the similarity rate is around 60-100\% (Figure 10). If the average of similarity rate is observed, the result is as tabulated in Table 2. From the average similarity rate compiled in Table 2, the Laplacian filter has higher ability to accurately detect the same vein pattern than Frangi-based filter. But in translating the vein pattern visually, both filtering techniques can detect the vein pattern in the NIR palm image in its own way.

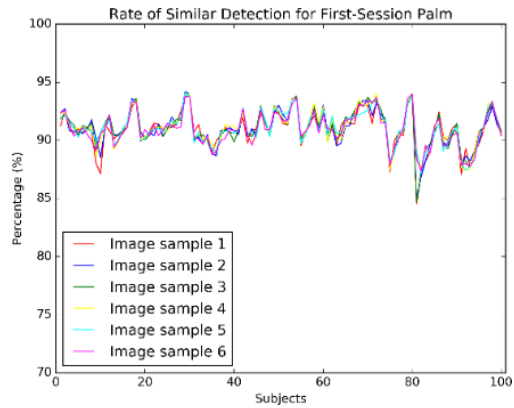

(a)

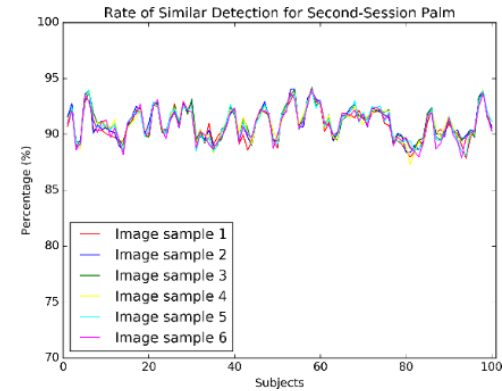

(b)

Figure 9. Rate of Similarity for Pixel-by-Pixel Detection of Polyu Database After Implementation of Laplacian Filter for (a) First-Session Palm, and (b) Second-Session Palm 


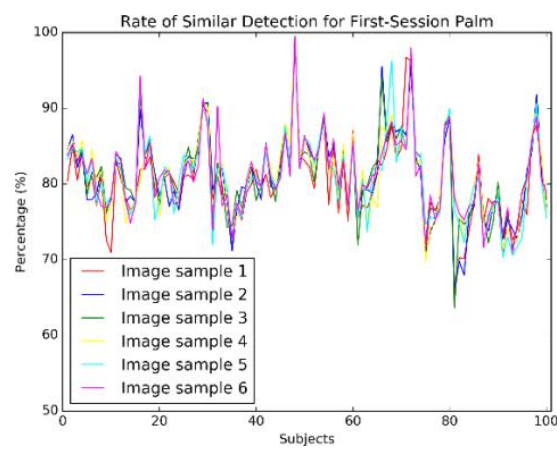

(a)

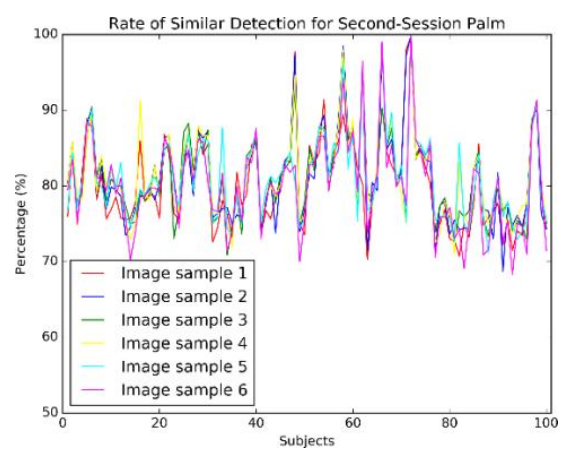

(b)

Figure 10. Rate of Similarity for Pixel-by-Pixel Detection of Polyu Database After Implementation of FrangiBased Filter for (a) First-Session Palm, and (b) Second-Session Palm

Table 2. Average Rate of Similarity Percentage for Pixel-by-Pixel Detection for Both Filtering Techniques

\begin{tabular}{cccc}
\hline Database & Hand/Session & Filtering Technique & Average Rate of Similar Detection (\%) \\
\hline CASIA & Right-hand & Laplacian & 95 \\
& & Frangi-based & 89 \\
& Left-hand & Laplacian & 96 \\
& & Frangi-based & 89 \\
\multirow{3}{*}{ PolyU } & First-session & Laplacian & 90 \\
& & Frangi-based & 80 \\
& Second-session & Laplacian & 91 \\
& & Frangi-based & 80 \\
\hline
\end{tabular}

\section{CONCLUSION}

Based on outcomes compiled in Section 3, both filtering techniques have the ability to detect palm vein pattern from a NIR palm image, but the difference lies in the type of vein pattern detected. While Laplacian filter detect vein pattern contributed by all vein sizes, Frangi-based filter detects only large-sized vein and highly affected by the illumination error acquired in the raw palm image. Even so, the implementation of Laplacian filter required additional image pre-processings to be executed on the raw palm image. With the image pre-processings, only then the Laplacian filter can accurately detects vein pattern in the image. In contrast, Frangi-based filter implementation does not need additional pre-processings to detect palm vein pattern in a NIR palm image. But the detection of vein pattern in Frangi-based filtered image are affected by illumination radiated during the acquisition process. Uneven illumination in the acquired image will give inaccurate extraction of vein pattern in the Frangi-based filtered image.

Depending on application, both filtering techniques have their own strengths and disadvantages. Translated vein pattern by Laplacian filter is more favourable for biometric application since it is rich in information that can be used as matching features. In areas where the visualization of vein pattern is more important, Frangi-based filtered image is just as sufficient. Perhaps the implementation of Frangi-based filter can be further improved so that the detected vein pattern is of the same size as its original vein pattern localized in the raw palm image. Improvement may includes implementation of necessary pre-processing or post-processing that can preserve the vein pattern size detected by the filtering technique. Other than that, image acquisition device and its process can be further enhanced, such that it can capture vein pattern information accurately without interference from the illumination used or other noises during the acquisition process.

With that, it is hoped that the findings presented in this paper can give some guidelines and ideas for researchers with the same interests to decide the appropriate filtering techniques for their intended vein pattern application.

\section{ACKNOWLEDGEMENTS}

Our deepest appreciation to the Ministry of Higher Education Malaysia, Universiti Putra Malaysia (UPM) and Universiti Teknikal Malaysia Melaka (UTeM) for all the facilities and support given during the duration of this study. 


\section{REFERENCES}

[1] M. A. Ahmed, H. M. Ebied, E. M. El-Horbaty, A. M. Salem, "Analysis of Palm Vein Pattern Recognition Algorithms and Systems," Journal of Bio-Medical Informatics and E-Health, vol. 1, no. 1, pp. 10-14, 2013.

[2] J.-C. Lee, “A Novel Biometric System Based on Palm Vein Image," Pattern Recognition Letters, vol. 33, no. 12, pp. 1520-1528, Sep. 2012.

[3] R. K. M, G. Deepika, M. Krishnan, B. Karthikeyan, "An Open Source Contact-Free Palm Vein Recognition System," International Journal of Advances in Applied Sciences (IJAAS), vol. 6, no. 4, pp. 319-324, 2017.

[4] S. Juric, B. Zalik, "An Innovative Approach to Near-Infrared Spectroscopy Using a Standard Mobile Device and Its Clinical Application in The Real-Time Visualization of Peripheral Veins," BMC Medical Informatics and Decision Making, vol. 14, no. 100, pp. 1-9, 2014.

[5] K. I. Ahmed, M. H. Habaebi, M. R. Islam, "Enhanced Vein Detection from Video Sequences, ” Indonesian Journal of Electrical Engineering and Computer Science (IJEECS), vol. 8, no. 2, pp. 420-427, 2017.

[6] W. Wu_a, F. Lu, G. Cheng, C. Shi, “A Vein Based Biometric Experiment and Some New Developments,” in 2012 Third Global Congress on Intelligent Systems, 2012, pp. 131-135.

[7] Z. Honarpisheh, K. Faez, "An Efficient Dorsal Hand Vein Recognition based on Firefly Algorithm," International Journal of Electrical and Computer Engineering (IJECE), vol. 3, no. 1, pp. 30-41, 2013.

[8] A. Pflug, D. Hartung, C. Busch, "Feature Extraction From Vein Images Using Spatial Information and Chain Codes," Information Security Technical Report, vol. 17, no. 1-2, pp. 26-35, Feb. 2012.

[9] L. Wang, G. Leedham, S.-Y. Cho, "Infrared Imaging of Hand Vein Patterns for Biometric Purposes," IET Computer Vision, vol. 1, no. 3, pp. 113-122, Dec. 2007.

[10] M. Soni, S. Gupta, M. S. Rao, P. Gupta, "An Efficient Vein Pattern-Based Recognition System," 2010 Fourth International Conference on Emerging Security Information, Systems and Technologies, pp. 234-239, Jul. 2010.

[11] D. Hartung, M. A. Olsen, H. Xu, C. Busch, "Spectral Minutiae for Vein Pattern Recognition," 2011 International Joint Conference on Biometrics (IJCB), pp. 1-7, Oct. 2011.

[12] R. D. Prasanna, P. Neelamegam, S. Sriram, N. Raju, "Enhancement of Vein Patterns in Hand Image for Biometric and Biomedical Application using Various Image Enhancement Techniques," Procedia Engineering, vol. 38, pp. 1174-1185, 2012.

[13] A. Shahzad, C. M. Goh, N. M. Saad, N. Walter, A. S. Malik, F. Meriaudeau, "Subcutaneous Veins Detection and Backprojection Method Using Frangi Vesselness Filter, ” in 2015 IEEE Symposium on Computer Applications \& Industrial Electronics (ISCAIE), 2015, pp. 65-68.

[14] R. Laganiere, OpenCV 2 Computer Vision Application Programming Cookbook. Packt Publishing, 2011.

[15] A. F. Frangi, W. J. Niessen, K. L. Vincken, M. A. Viergever, "Multiscale Vessel Enhancement Filtering," International Conference on Medical Image Computing \& Computer-Assisted Intervention. Springer Berlin Heidelberg, pp. 130-137, 1998.

[16] OpenCV Development Team, “OpenCV Documentation,” 2015. [Online]. Available: http://docs.opencv.org/.

[17] CASIA, "CASIA-MS-PalmprintV1,” 2014. [Online]. Available: http://biometrics.idealtest.org.

[18] PolyU, "PolyU Multispectral Palmprint Database," 2014. [Online]. Available: http://www.comp.polyu.edu.hk/ biometrics/MultispectralPalmprint/MSP.htm. 


\section{BIOGRAPHIES OF AUTHORS}
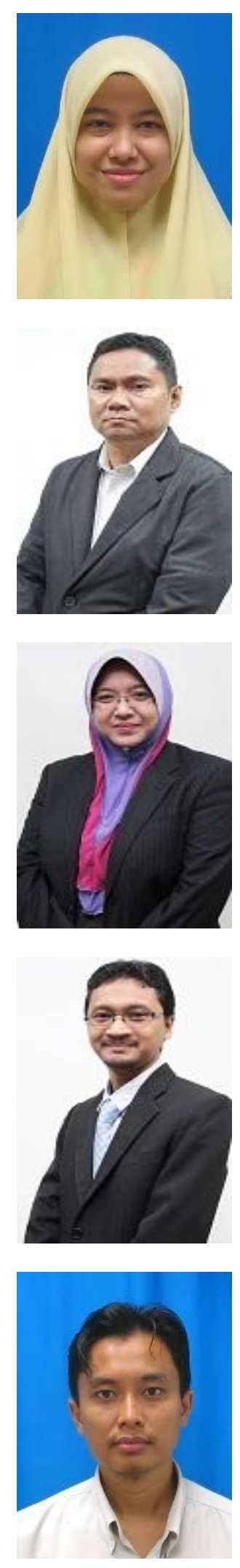

Zarina Mohd Noh received her MEng degree in electrical from the Universiti Teknologi Malaysia (UTM) in 2009 and since then has been attached to the Universiti Teknikal Malaysia Melaka (UTeM). She is currently a Ph.D candidate at the Faculty of Engineering in the Universiti Putra Malaysia (UPM). Her main research interests are in the field of computer and embedded system engineering.

Abdul Rahman Ramli received his Ph.D from the University of Bradford, UK in 1995. He is currently an Associate Professor at the Department of Computer and Communication Systems Engineering, Faculty of Engineering, Universiti Putra Malaysia (UPM). He was the Head of Intelligent System and Robotic Laboratory (ISRL) at the Institute of Advanced Technology, UPM from 2002 to 2011. To date, he has more than 200 journals and conference papers published. His research interests include embedded and real-time system, image processing and multimedia system.

Marsyita Hanafi received her Ph.D from the Imperial College London in 2012. She has been employed by the Universiti Putra Malaysia (UPM) since 2000 and is currently a Senior Lecturer at the Department of Computer and Communication Systems Engineering, Faculty of Engineering, UPM. Her research interests include digital image processing, pattern recognition, security and biometric.

M. Iqbal Saripan received his Ph.D from the University of Surrey, UK in the area of image processing. He is currently a Professor at the Department of Computer and Communication Systems Engineering, Faculty of Engineering, Universiti Putra Malaysia (UPM). He is also a member of the IEEE and the Institute of Physics UK. His research area is in image processing, particularly in medical imaging.

Ridza Azri Ramlee received his Master (2008) in Telecommunication and Information Engineering from the Universiti Teknologi MARA (UiTM) and BEng (2000) in Electrical Engineering from the Universiti Teknologi MARA (UiTM), Malaysia. He is also a cooperate member of Institute of Engineer Malaysia (IEM), graduate member of IEEE and Professional Engineer granted by Board of Engineering Malaysia (BEM) in 2012. He is currently pursuing his Ph.D at the Universiti Putra Malaysia (UPM) in the field of Communication and Network Engineering. His research interests are in the area of image processing, industrial electronic instrumentation and electronic embedded system. 